\title{
Critical state in thin anisotropic superconductors of arbitrary shape
}

\author{
Grigorii P. Mikitik \\ B. Verkin Institute for Low Temperature Physics 86 Engineering, National Ukrainian Academy of Sciences, Kharkov 310164, \\ Ukraine \\ Ernst Helmut Brandt \\ Max-Planck-Institut für Metallforschung, D-70506 Stuttgart, Germany
}

(October 27, 2018)

\begin{abstract}
A thin flat superconductor of arbitrary shape and with arbitrary in-plane and out-of-plane anisotropy of flux-line pinning is considered, in an external magnetic field normal to its plane. It is shown that the general three-dimensional critical state problem for this superconductor reduces to the two-dimensional problem of an infinitely thin sample of the same shape but with a modified induction dependence of the critical sheet current. The methods of solving the latter problem are well-known. This finding thus enables one to study the critical states in realistic samples of high- $T_{c}$ superconductors with various types of anisotropic flux-line pinning. As examples, we investigate the critical states of long strips and rectangular platelets of high- $T_{c}$ superconductors with pinning either by the $a b$ planes or by extended defects aligned with the $c$ axis.
\end{abstract}

PACS numbers: 74.60.-w, 74.60.Ge, 74.60.Jg

\section{INTRODUCTION}

Numerous publications deal with the critical state problem in isotropic thin superconductors placed in a perpendicular magnetic field.1 By thin we mean that the sample thickness $d$ is considerably less than its lateral extension. Analytic solutions of the problen were obtained in the cases of a circular disk2, a strip 3, and elliptic-shaped films. 4 For thin superconductors of an arbitrary shape, numerical methods for solving this problem were elaborated in Ref. 5,5, and the critical state of a rectangular platelet was investigated in detail in Ref. [7. All the above-mentioned results can be derived considering the appropriate flat superconductors to be infinitely thin. In the framework of this approach a possible in plane anisotropy of pinning can be taken into account a, 8 yielding good agreement with appropriate magnetooptic experiments. 8

It is important to note that in the highly anisotropic high- $T_{c}$ superconductors the flux-line pinning in general depends on the angle $\theta$ between the local direction of the magnetic induction $\mathbf{B}$ and the $c$ axis which in typical experiments is normal to the plane of the samples. For example, this type of anisotropy occurs when one takes into account the intrinsic pinning exerted by the $\mathrm{CuO}$ planes 9 Besides this, twin boundaries, columnar defects, and other extended defects give rise to such an out-ofplane anisotropy. In all these cases, the flux-line curvature that always occurs in thin superconductors placed in a perpendicular magnetic field, leads to a dependence of the critical current density $j_{c}$ on the coordinate $z$ across the thickness of the sample. Therefore, the critical state problem becomes three-dimensional (3D), and the feasibility of treating such superconductors as infinitely thin requires special consideration.

For a thin anisotropic disk with radius $R$ placed in a perpendicular magnetic field it was shown 10 that the small ratio $d / R$ enables one to split the $2 \mathrm{D}$ critical state problem into two one-dimensional problems. The first one treats the critical state across the thickness of the disk, while the second one treats the disk as infinitely thin and gives the distributions of magnetic field and sheet current along its radius. Using this method, the magnetic moment of a thin anisotropic disk was theoretically studied 11 in the case when the angular dependent $j_{c}$ has a peak at $\theta=0$. In this special geometry of the circular disk the directions of field and current are fixed and known in advance, and the critical state problem thus is essentially not $3 \mathrm{D}$ but only $2 \mathrm{D}$. This simplification does not take place in thin samples of arbitrary shape, where the current stream lines, magnetic contour lines, and penetrating flux fronts in general do not coincide. 1 Moreover, according to Ref. 4 , in the general case the directions of the circulating currents may change across the thickness of the sample, and a rotation or twist of the flux line arrangement is possible.

In the present paper we show that even in the general $3 \mathrm{D}$ critical state problem of a thin superconductor with arbitrary shape and arbitrary anisotropy of $j_{c}$, the $3 \mathrm{D}$ pinning problem can be split into two simpler problems which can be solved: The 1D problem across the thickness of the sample is solved analytically, while the inplane problem reduces to that of the infinitely thin superconductor which was treated before. Since the methods of solving the latter problem are well elaborated, 5 our finding enables one to study the critical state in flat samples of arbitrary shape and with any type of anisotropy. As concrete examples, we shall investigate the critical states of long strips and rectangular platelets with various types of out-of-plane anisotropy. 

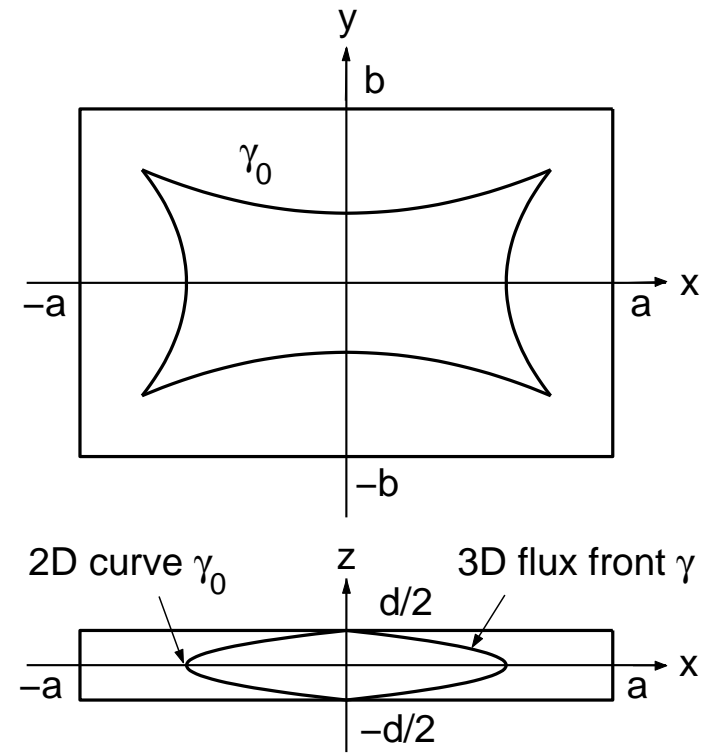

FIG. 1. The front of magnetic flux penetrating into a thin rectangular superconductor plate with pinning when the applied perpendicular magnetic field increases from zero. The top figure shows the two-dimensional curve $\gamma_{0}$ that forms the equator of the three-dimensional flux front $\gamma$ shown in the lower plot. Inside this $3 \mathrm{D}$ front the magnetic induction $\mathbf{B}$ and current density $\mathbf{j}$ are exactly zero, and inside the curve $\gamma_{0}$ the perpendicular component $B_{z}$ is practically zero when $d \ll(a, b)$ for a thin plate with dimensions $2 a \times 2 b \times d$.

\section{SPLITTING THE 3D PROBLEM}

In what follows we assume that the pinning-caused characteristic magnetic field $H_{c r}=j_{c} d / 2$ considerably exceeds the lower critical field $H_{c 1}$, and the thickness $d$ of the sample is much greater than the London penetration depth $\lambda$. Under these conditions the magnetic induction is practically equal to the magnetic field in the superconductor, and the so-called geometrical barrier 12 is negligible. We study the critical state problem in the framework of the macroscopic approach, in which all considered lengths are larger than the flux-line spacing, and treat the superconductor as a uniform anisotropic medium.

Let us place the coordinate system so that its $x y$ plane coincides with the middle plane of the sample and the $z$ axis is directed along the external magnetic field; the case of a rectangular platelet is shown in Fig. 1. Since the thickness $d$ of the sample is assumed to be much less than its smallest lateral dimension $L$, the strict equations $\nabla \cdot \mathbf{j}=0$ and $\nabla \cdot \mathbf{B}=0$ give that, in leading order in the parameter $d / L$, one has $j_{z}=0$, and the perpendicular field component $B_{z}$ is independent of $z$ inside the sample, i.e., $B_{z}=B_{z}(x, y)$. Accounting for the fact that the scale of changes with $x$ and $y$ of $B_{z}$ and of the in-plane field $\mathbf{B}_{t}=\left(B_{x}, B_{y}\right)$ is $L$, while the appropriate scale for changes of $\mathbf{B}_{t}$ across the thickness is $d$, we may neglect the derivatives of $\mathbf{B}$ with respect to $x$ and $y$. The critical state equation can thus be written in the form:

$$
\hat{\mathbf{z}} \times \frac{\partial \mathbf{B}_{t}}{\partial z}=\mu_{0} \mathbf{j}_{c},
$$

where $\hat{\mathbf{z}}$ is the unit vector along $z$ and $\mathbf{j}_{c}$ is the critical current density. This equation means that the critical gradient develops predominantly across the thickness of the sample, and we can consider the fields and the currents to be independent of $x, y$ in sufficiently small parts of the sample with dimensions in the $x, y$ directions much greater than $d$ and much less than $L$. To proceed our analysis we write $\mathbf{B}_{t}=B_{t} \hat{\mathbf{t}}$ and $\mathbf{j}_{c}=j_{c} \hat{\mathbf{n}}$, where $\hat{\mathbf{n}}=(\cos \varphi, \quad \sin \varphi)$ and $\hat{\mathbf{t}}=(\cos \psi, \sin \psi)$. The angles $\varphi(x, y, z)$ and $\psi(x, y, z)$ define the directions of $\mathbf{j}_{c}$ and $\mathbf{B}_{t}$ in $x y$ planes. The above critical state equation can then be rewritten as

$$
\begin{gathered}
\frac{\partial B_{t}}{\partial z}=\mu_{0} j_{c} \sin (\varphi-\psi), \\
B_{t} \frac{\partial \psi}{\partial z}=-\mu_{0} j_{c} \cos (\varphi-\psi) .
\end{gathered}
$$

We emphasize that in the general case the critical current density $j_{c}$ may depend on $B_{z}, B_{t}, \varphi-\psi$, $\psi$, i.e., $j_{c}=j_{c}\left(B_{z}, B_{t}, \varphi-\psi, \psi\right)$. The quantities $B_{t}$ and $B_{z}$ can be also expressed in terms of the magnitude $B \equiv|\mathbf{B}|$ and the tilt angle $\theta$ between the local direction of $\mathbf{B}$ and the $\mathrm{z}$ axis:

$$
B_{z}=B \cos \theta, \quad B_{t}=B \sin \theta .
$$

The dependence on $\psi$ occurs only if there is an anisotropy of pinning in the $x$ yplane (e.g., when twin boundaries exist in the sample 13 ). In the isotropic case, when the flux-line pinning depends neither on $\psi$ nor on the tilt angle $\theta$, the dependence of $j_{c}$ on $B_{z}$ and $B_{t}$ occurs only through the magnitude $B$. Note also that, since $B_{z}$ is independent of $z$, it enters Eqs. $(1,2)$ as a parameter, and thus we have only two equations for the three functions $B_{t}, \varphi$, and $\psi$. One more equation can be obtained if one takes into account the prehistory of the critical state, telling how the flux lines were pushed into the sample.

In increasing moderate magnetic field there is a flux and current-free core in the superconductor, Fig. 1. The surface of the core, $\gamma$, is the penetrating flux front, which we describe by a function $z=z_{\gamma}(x, y)$. In the limiting case of an infinitely thin superconductor $(d \rightarrow 0)$ the flux front may be thought of as a flat curve $\gamma_{0}$, forming the outer rim (equator) of $\gamma$, since we have $B_{z}=0$ inside this contour. In the case of small but finite $d \ll L$, the component $B_{z}$ is still practically zero in the region inside $\gamma_{0}$ since there the flux lines are almost parallel to the flat surfaces of the sample. Note that these flux lines may have different orientations at different values of $z$ if the sample is not a circular disk or a strip; thus, in principle, $\mathrm{H}_{y}$-cutting processes may occur in the superconductor. $14-16$

We now consider the third required equation in the region $z_{\gamma}(x, y) \leq|z| \leq d / 2$ with $x, y$ inside the curve $\gamma_{0}$, 
i.e. between the lens-shaped empty core and the flat surfaces. In increasing applied field the flux lines are pushed continuously from the flat surfaces into this region, such that the in-plane orientation of the flux lines is a unique function of $B_{t}, x$, and $y$ :

$$
\psi=\Psi\left(B_{t}, x, y\right) .
$$

The function $\Psi$ is found from the solution of the critical state problem for the infinitely thin sample (see below). Equation (3) is just the third required equation. Now we are able to solve Eqs. (1-3). Dividing Eq. (2) by Eq. (1) we obtain

$$
B_{t} \frac{\partial \psi}{\partial B_{t}}=-\cot (\varphi-\psi) .
$$

Inserting here the known field orientation, Eq. (3), we find the difference $\varphi-\psi$ and the angle of the currents $\varphi$ as functions of $B_{t}$. Finally, the dependence of $B_{t}$ on $z$ can be obtained from the implicit relation

$$
z-z_{\gamma}=\int_{0}^{B_{t}} \frac{d b_{t}}{\mu_{0} j_{c}\left(0, b_{t}, \pi / 2, \Psi\left(b_{t}, x, y\right)\right)},
$$

which follows from Eq. (1), and from the formula

$$
j_{c}\left(0, B_{t}, \varphi-\psi, \psi\right)=\frac{j_{c}\left(0, B_{t}, \pi / 2, \psi\right)}{\sin (\varphi-\psi)},
$$

reflecting the fact that only the component of $j_{c}$ normal to $\mathbf{B}$ is essential (in absence of flux cutting). The function $z_{\gamma}(x, y)$ describing the shape of the flux front is obtained from the equality:

$$
\frac{d}{2}-z_{\gamma}=\int_{0}^{B_{s}} \frac{d b_{t}}{\mu_{0} j_{c}\left(0, b_{t}, \pi / 2, \Psi\left(b_{t}, x, y\right)\right)},
$$

where $B_{s}(x, y)=B_{t}(x, y, d / 2)$ is the tangential component of the surface field. This $B_{s}$ may be obtained by solving the critical state equation for infinitely thin superconductors. Thus, Eqs. (3-6) give the solution of the critical state problem across the thickness of the sample in the region inside the curve $\gamma_{0}$.

It should be emphasized that the above formula for the dependence of $j_{c}$ on $\varphi-\psi$ and Eqs. $(3,5,6)$ hold only if flux cutting does not occur in the superconductor when the critical state is established in it. Flux cutting will only happen if $|\cot (\varphi-\psi)|$, calculated from Eq. $(4)$, turns out to be greater than some critical value $\chi 15$ In this case the so-called generalized critical-state mode 15 must be used to describe the critical state in the region of the sample inside $\gamma_{0}$. However, since at present we cannot point out the shape of the superconductor for which this flux cutting really occurs, we do not analyze this case here.

Let us now derive the third equation for the region where $B_{z} \neq 0$, i.e., outside the curve $\gamma_{0}$. In deriving it is necessary to take into account that in an anisotropic superconductor the direction of the flux-line velocity in general does not coincide with the direction of the Lorentz force causing this movement, and thus the appropriate electric field $\mathbf{E}$ is not always parallel to the current density (see Appendix A). With this in mind, the third equation results from the following two conditions: First, the shape of a flux line cannot change when an applied magnetic field $H$ increases slightly so that $\gamma_{0}$ and all the flux lines in this region of the sample are shifted by distances which are much less than the sample width $L$. Second, during the shift, each line element moves along the local direction of $[\mathbf{E} \times \mathbf{B}]$. The geometrical analysis of these conditions gives

$$
\frac{B_{z}^{2} \sin \xi-B_{t}^{2} \cos \psi \sin (\psi-\xi)}{B_{z}^{2} \cos \xi+B_{t}^{2} \sin \psi \sin (\psi-\xi)}=\mathrm{const}
$$

where the angle $\xi$ is determined by Eqs. $(\mathrm{A} 2, \mathrm{~A} 3, \mathrm{~A} 5)$ in Appendix A. Since $B_{t}(0)=0$, the obtained relation can be rewritten as

$$
\tan [\xi-\xi(0)]=\frac{B_{t}^{2} \sin [\psi-\xi(0)] \cos [\psi-\xi(0)]}{B_{t}^{2} \cos ^{2}[\psi-\xi(0)]+B_{z}^{2}},
$$

with $\xi(0) \equiv \xi(x, y, 0)$. This is the third required equation in the region where $B_{z} \neq 0$. When an in-plane anisotropy of the flux line pinning is absent, it is possible to find the solution of Eqs. $(1,2,7)$ in the interval $0 \leq z \leq d / 2$. The initial conditions to these equations are

$$
B_{t}(0)=0, \quad \varphi(0)-\psi(0)=\pi / 2,
$$

where the last equality follows from Eq. (2) with $B_{t}(0)=$ 0 . Since the solution of the equations is unique, it is sufficient to guess it. The solution has the form

$$
\begin{array}{r}
\varphi(x, y, z)=\varphi_{0}(x, y), \\
\psi(x, y, z)=\varphi_{0}(x, y)-\pi / 2 \equiv \psi_{0}(x, y), \\
z=\int_{0}^{B_{t}} \frac{d b_{t}}{\mu_{0} j_{c}\left(B_{z}, b_{t}, \pi / 2, \psi_{0}\right)} .
\end{array}
$$

Here the angle $\varphi_{0}(x, y)$ defines the direction of the sheet current in the critical state of the infinitely thin superconductor and is assumed to be known. In obtaining the solution we have taken into account that $\xi=\varphi$ at $\varphi-\psi=\pi / 2$ when the in-plane anisotropy is absent. Eqs. (9-11) mean that the flux lines are curved in the region where $B_{z} \neq 0$, but they are not twisted there. In other words, the distributions of the magnetic induction and the current across the thickness of the sample are the same as in a circular disk or a strip if one considers small regions in the $x y$ plane with the same values of $B_{z}$. Eq. (11) allows us to find an implicit relation between the sheet current $J$ and $B_{z}$ :

$$
\frac{d}{2}=\int_{0}^{J / 2} \frac{d h_{t}}{j_{c}\left(B_{z}, \mu_{0} h_{t}, \pi / 2, \psi_{0}\right)} .
$$


Here we have used that $B_{t}(z=d / 2) \equiv B_{s}=\mu_{0} J / 2$, where $J \equiv|\mathbf{J}|$ and $\mathbf{J}$ is the current density integrated over the film thickness. It should be noted that although we indicate explicitly the angle $\psi_{0}$ as an argument of $j_{c}$ in Eqs. (11,12), the critical current density is independent of this angle in cases without in-plane anisotropy.

We now discuss the case when some in-plane anisotropy of flux-line pinning exists in the superconductor together with the out-of-plane anisotropy, i.e. we allow for a dependence of $j_{c}$ on $\psi$. This case can occur, e.g. in twinned crystals of high- $T_{c}$ superconductors. When an in-plane anisotropy exists, it is impossible to find the solution of Eqs. $(1,2,7)$ in the general form. We may only state that in this situation the in-plane angle $\psi$ generally depends on $z$, and thus flux lines are not only curved but also twisted. Then, according to Eq. (2), the current is not normal to the magnetic induction. The magnitude of the twist is determined by the relative strengths of the in-plane and out-of-plane anisotropies (and, of course, by the thickness of the sample). Interestingly, when $j_{c}$ does not depend on $B_{t}$, the solution described by Eqs. (9-11) becomes true again, and the twist disappears. (This situation occurs, e.g., in sufficiently large magnetic fields, $H \gg H_{c r}$, or if there is no out-of-plane anisotropy in the superconductor and $j_{c}$ is practically independent of the magnitude of the magnetic induction). However, if $H$ changes, in this case a flux line is shifted in a direction which does not lie in the plane containing the line. According to the results of Appendix A, the direction makes an angle $\alpha$ with the above-mentioned plane where

$$
\tan \alpha=-\left.\frac{\partial\left[\ln j_{c}\left(B_{z}, \varphi-\psi_{0}, \psi_{0}\right)\right]}{\partial \varphi}\right|_{\varphi=\psi_{0}+\frac{\pi}{2}}
$$

To sum up, it is important to emphasize that at a given dependence of $j_{c}$ on $B_{z}, B_{t}, \varphi-\psi$ and $\psi$, the onedimensional equations (1), (2), (7) with initial conditions (8) can be solved (at least numerically) in the case of an arbitrary anisotropy of flux-line pinning, and a relation $J\left(B_{z}, \varphi_{0}\right)$ generalizing Eq. (12) can be obtained. Here $\varphi_{0}$ defines the direction of the sheet current in the $x y$ plane.

Thus, we have reduced the $3 \mathrm{D}$ critical state problem to a $2 \mathrm{D}$ problem in which the $B_{z}$-dependence of the sheet current $J\left(B_{z}, \varphi_{0}\right)$ is determined not only by the $B_{z}$-dependence of the critical current density $j_{c}$ but also by its dependence on the tilt angle $\theta$ of the flux lines away from the film normal, see Eq. (12). It should be noted, however, that to obtain this reduction we have assumed that $d$ is much less than the characteristic scale of changes of $\mathbf{B}$ in the $x y$ plane. Numerical solutions of the critical state equation for infinitely thin superconductors of various shapes show that this assumption indeed is true everywhere; the scale of variation is of the order of $L$ except for a small region near the flux front $\gamma_{0}$ where the derivatives $\partial B_{x} / \partial y$ and $\partial B_{y} / \partial x$ are not small compared with $\partial \mathbf{B}_{t} / \partial z$. However, the width of this region is determined by the thickness $d$ and is thus small.

Knowing the function $J\left(B_{z}, \varphi_{0}\right)$ obtained from
Eq. (12) or from the above-mentioned generalization of this equation, it is possible to solve the critical state equatipns for an infinitely thin superconductor of any shape.4.5 The static equations to be solved are

$$
\nabla \cdot \mathbf{J}=0
$$

and the Biot-Savart law connecting $B_{z}$ with the sheet current $\mathbf{J}(x, y)=\int_{-d / 2}^{d / 2} \mathbf{j}(x, y, z) d z$,

$$
\mu_{0}^{-1} B_{z}(\mathbf{r})=H+\int_{S} \frac{[\mathbf{R} \times \mathbf{J}]}{4 \pi R^{3}} d^{2} r^{\prime},
$$

where $\mathbf{R}=\mathbf{r}-\mathbf{r}^{\prime} ; \mathbf{r}$ and $\mathbf{r}^{\prime}$ are $2 \mathrm{D}$ vectors in the $x y$ plane, and the integration is carried out over the specimen area $S$. In addition, one has to take into account that $B_{z}=0$ inside the 2D flux front $\gamma_{0}$, while outside $\gamma_{0}$ the sheet current is equal to $J\left(B_{z}, \varphi_{0}\right)$. At given applied field $H$, a solution of this static problem exists only for a certain curve $\gamma_{0}$, which determines the shape and position of the penetrating flux front. In Refs. 1,5, 7, in fact, the more general dynamic equations were considered, which allow one to find the solution of the 2D critical state equations very effectively for any prehistory $H(t)$. The appropriate formulas are presented in Appendix B.

Solving these equations for the infinitely thin superconductor of arbitrary shape in a perpendicular applied field $H$, one obtains $B_{z}(x, y, H), J(x, y, H), \varphi_{0}(x, y, H)$, $B_{s}(x, y, H)=\mu_{0} J / 2$, the direction of $\mathbf{B}_{s}$, i.e., the function $\psi_{0}(x, y, H)=\varphi_{0}(x, y, H)-\pi / 2$, and also the position of $\gamma_{0}$. Eliminating $H$ from $\psi_{0}(x, y, H)$ and $B_{s}(x, y, H)$ in the region inside $\gamma_{0}$, and replacing $B_{s}$ by $B_{t}$ we arrive at the function $\Psi\left(B_{t}, x, y\right)$ that enters Eq. (3). Thus, the solution of the $2 \mathrm{D}$ critical state problem for the infinitely thin superconductor enables one to obtain all the necessary information for the calculations of the core shape $z_{\gamma}(x, y)$ and distributions of $B_{t}, \psi, \varphi$ across $z$ at any $x$ and $y$. The knowledge of all these functions permits to describe the $3 \mathrm{D}$ critical state of thin anisotropic superconductors in detail.

\section{OUT-OF-PLANE ANISOTROPY}

We now use the results obtained above to investigate the critical states of thin superconductors with out-ofplane anisotropy alone. That is, we consider the case when the critical current density $j_{c}\left(B_{z}, B_{t}, \pi / 2, \psi\right)$ does not depend on the in-plane angle $\psi$. In addition we here neglect its dependence on $B$ such that $j_{c}$ depends only on the flux-line tilt angle $\theta, j_{c}=j_{c}(\theta)$. This situation occurs when the flux-line pinning is isotropic in the $x y$ plane, and the scale $B_{0}$ characterizing the dependence of $j_{c}$ on $B$ considerably exceeds the self-fields of the critical currents. As shown above, the anisotropy of $j_{c}(\theta)$ can be accounted for by introducing an appropriate $B_{z}$ dependence of the sheet current $J=J\left(B_{z}\right)$, implicitly given by 

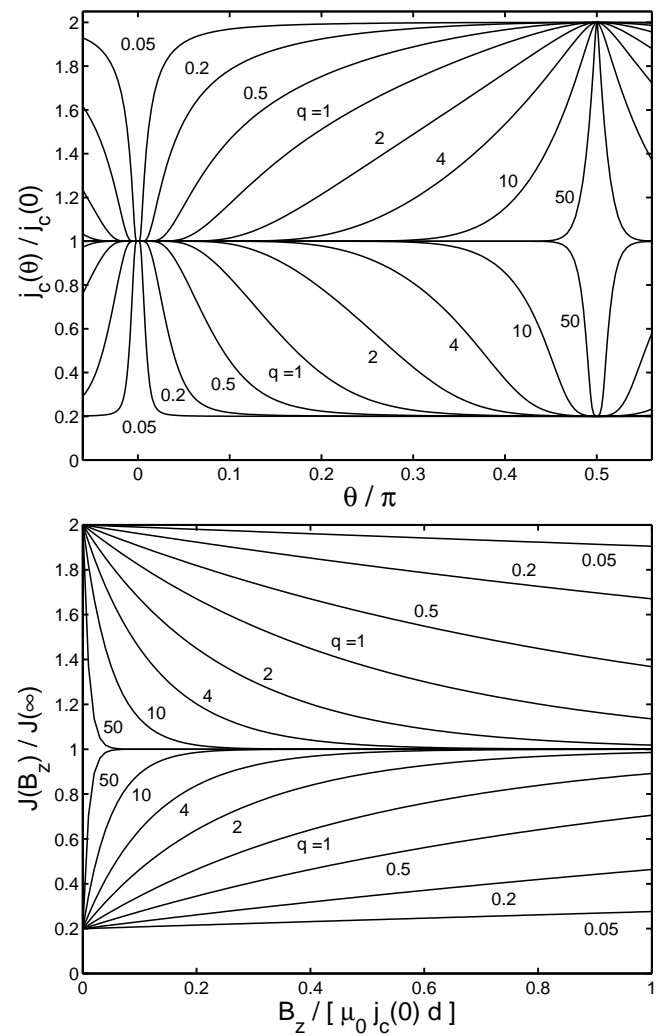

FIG. 2. The out-of-plane anisotropy of the critical current density $j_{c}(\theta)$ described by the model (18) (top plot) and the equivalent induction dependence of the sheet current $J\left(B_{z}\right)$ of Eq. (17) (lower plot). In both plots the upper half is for the parameter $p=1$ and the lower half for $p=-0.8$. The second parameter is $q=0.05 \ldots 50$.

Eq. (12). In the considered case, a more explicit form of this dependence may be obtained in the following way. Differentiation of Eq. (12) with respect to $B_{z}$ yields the two equations:

$$
\begin{array}{r}
j_{c}(\theta) d=J\left(B_{z}\right)-B_{z} \frac{d J\left(B_{z}\right)}{d B_{z}}, \\
\tan \theta=\frac{\mu_{0} J\left(B_{z}\right)}{2 B_{z}} .
\end{array}
$$

In fact, Eqs. $(15,16)$ are the parametric form of that function $j_{c}(\theta)$ which results in a given dependence $J\left(B_{z}\right)$ via Eq. (12).

To fix ideas, we consider the following rather general model dependence for $J\left(B_{z}\right)$ :

$$
J\left(B_{z}\right)=j_{c}(0) d\left[1+p \exp \left(-\frac{q B_{z}}{B_{c r}}\right)\right],
$$

where $B_{c r}=\mu_{0} j_{c}(0) d / 2$, while $j_{c}(0), p$ and $q$ are the parameters of this example ( $p$ and $q$ are dimensionless). One then easily verifies that the corresponding angular dependence of the critical current density takes the form:

$$
\begin{array}{r}
j_{c}(\theta)=j_{c}(0)[1+p(1+q t) \exp (-q t)], \\
\tan \theta=t^{-1}[1+p \exp (-q t)],
\end{array}
$$

where $t$ is a curve parameter with range $0 \leq t \leq \infty$ equivalent to $\pi / 2 \geq \theta \geq 0$. This model dependence $j_{c}(\theta)$ is presented in Fig. 2 together with the corresponding $J\left(B_{z}\right)$, Eq. (17). For appropriate choices of the parameters $p$ and $q$ this model describes the intrinsic pinning by the $\mathrm{CuO}$ planes in high- $T_{c}$ superconductors $(p>0$, maximum $j_{c}$ at $\theta=\pi / 2$ ) and pinning by columnar defects perpendicular to the film $\left(p<0\right.$, maximum $j_{c}$ at $\theta=0$ ). It may thus be used to simulate these important cases. We remark that the critical state of a circular disk with some model for intrinsic pinning was studied numerically in Ref. 17, while the magnetization of the disk in the case when $j_{c}$ has a peak at $\theta=0$ was analyzed in Ref. 11. However, in those papers only the fully penetrated critical state was considered.

Below we calculate the partially and fully penetrated critical states in strips and rectangular platelets with anisotropic pinning using the angular dependence $j_{c}(\theta)$ described by Eqs. (18). The corresponding results for circular disks are verysimilar to the results for the strip. In fact the algorithm 18 used below to time-integrate the 1D equation of motion for the sheet current $f(x, t)$ in thin strips is almost identical to the algorithm 19 used for thin disks.

\section{THIN STRIP}

We begin with the calculation of the critical state in an infinitely thin strip (see Appendix B). In this calculation the function $J\left(B_{z}\right)$ described by Eq. (17) is considered as the critical value of the sheet current. The obtained results are presented in Figs. 3-7.

Figure 3 shows the induction profiles $B_{z}(x)$ and sheet current profiles $J(x)$ in increasing $H$ for thin strips with various anisotropies of $j_{c}(\theta)$ depending on the parameters $p$ and $q$. The profiles are presented for several values of $x_{0}$, the position of the $2 \mathrm{D}$ flux front $\gamma_{0}$. The reference case of isotropic pinning $(p=0)$ is also shown here. In Fig. 3a one can see that for $p>0$ (pinning by the $\mathrm{CuO}$ layers) the penetrating flux-density profile is very steep at $x=x_{0}: B_{z}(x)$ jumps almost abruptly and $J(x)$ has a very sharp peak which ideally should reach the maximum value $J\left(x_{0}\right)=(1+p) j_{c}(0) d$, cf. Eq. (17). In our computation this ideal maximum height is not reached due to our finite creep exponent $n=101$ and finite number $N=300$ of equidistant grid points (to illustrate the influence of $n$ the case of a small value $n=11$ is shown in Fig. 3d). As opposed to this, in Figs. $3 \mathrm{~b}$ and $3 \mathrm{c}$ the induction profiles for $p<0$ (pinning by linear defects perpendicular to the strip plane) are less sharp: at $x=x_{0}, B_{z}(x)$ vanishes less steeply (almost linearly for Fig. 3b) and $J(x)$ decreases monotonically and exhibits an inflection point with infinite slope, $d J /\left.d x\right|_{x=x_{0}}=\infty, J\left(x_{0}\right)=(1+p) j_{c}(0) d$. These general features of the flux front of superconductors with 

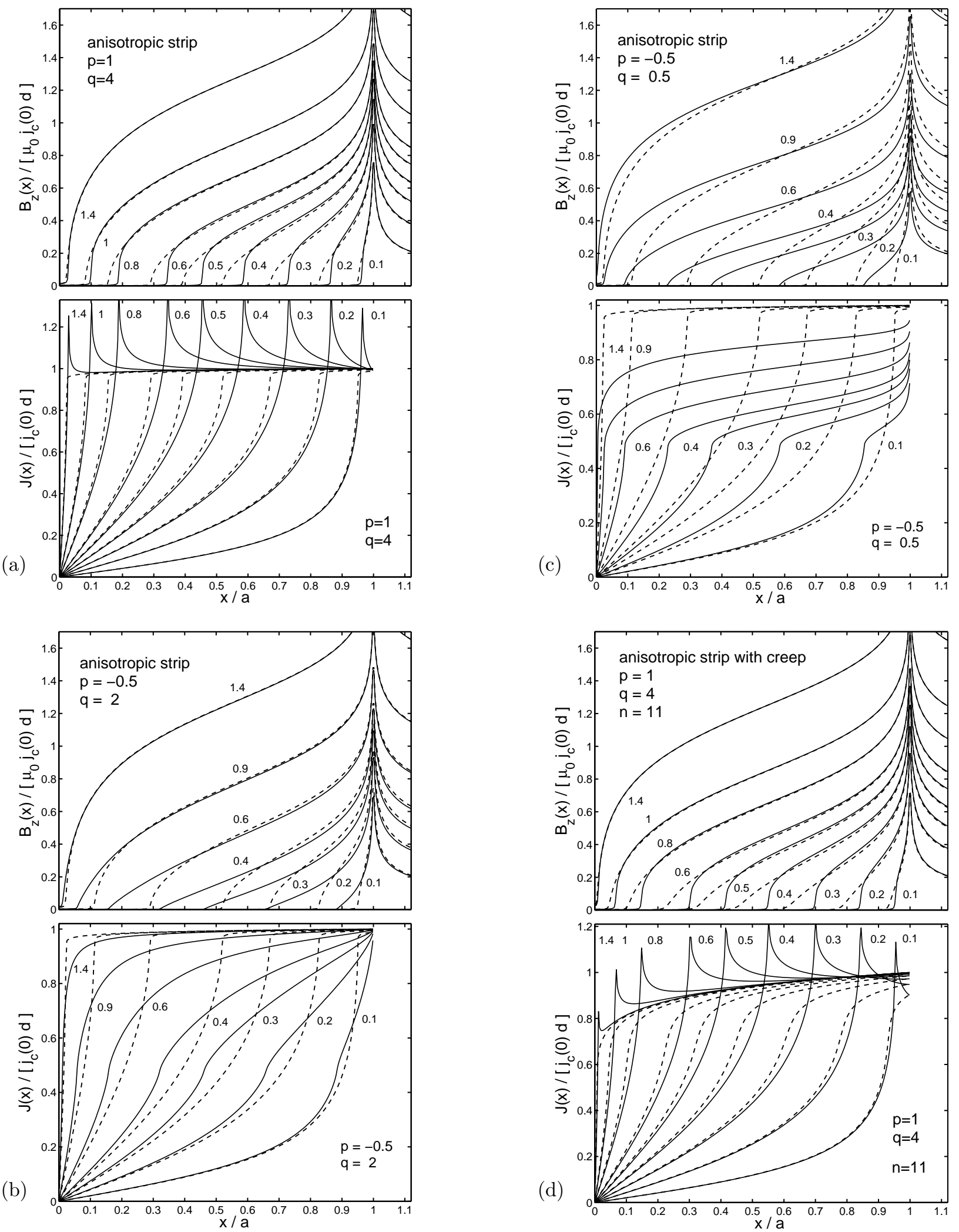

FIG. 3. Spatial profiles of the perpendicular field component $B_{z}(x)$ (upper plot) and of the sheet current $J(x)$ (lower plot) of a thin strip with anisotropic pinning described by the model (18), see Fig. 2. The various curves correspond to increasing applied field $H=0.1,0.2, \ldots 0.8,1,1.4$ in units of $j_{c}(0) d$. The dashed curves are for isotropic pinning $(p=0)$ and the solid curves for anisotropic pinning with parameters: a) $p=1, q=4$. b) $p=-0.5, q=2$. c) $p=-0.5, q=0.5$. Creep exponent $n=101$. To check the influence of $n$, the same case as in a) is shown in d) for $n=11$. 


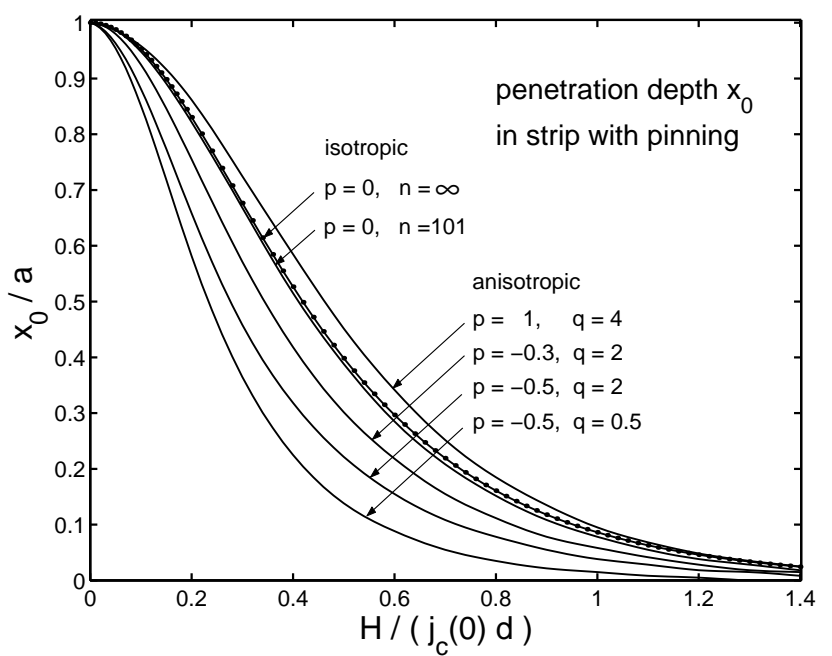

FIG. 4. The $x$ coordinate $x_{0}(H)$ of the penetrating flux front, or the penetration width $a-x_{0}$, for thin strips with various anisotropies of pinning $j_{c}(\theta)$, Eqs. (18), calculated with large creep exponent $n=101$. The line marked by dots shows the static analytical result for isotropic strips, Eq. (20).

anisotropic pinming are derived analytically in a forthcoming paper.20 It turns out that in the vicinity of $x_{0}$ the profiles are $B_{z}(x) \propto\left(x-x_{0}\right)^{\beta}$ and $J(x)-J\left(x_{0}\right) \propto\left|x-x_{0}\right|^{\beta}$ where $\beta \approx 0.5-\pi^{-1} \arctan (p q)$.

In the isotropic case $(p=0)$ our numerical resultspractically completely coincide with the exact solution ${ }^{3}$

$$
\begin{array}{r}
\frac{J(x)}{j_{c} d}=\frac{2}{\pi} \operatorname{arccot}\left\{\left[\max \left(0, \frac{\left(x_{0}^{2}-x^{2}\right) a^{2}}{\left(a^{2}-x_{0}^{2}\right) x^{2}}\right)\right]^{1 / 2}\right\}, \\
x_{0}=\frac{a}{\cosh \left(\pi H / j_{c} d\right)} .
\end{array}
$$

where $0 \leq x \leq a$ and $2 a$ is the width of the strip. It should be also mentioned that in the case of monotonically decreasing $J_{c}\left(B_{z}\right)$ the critical states of a thin strip and a thin disk were studied in Refs. 21, 22, respectively. In Ref. 21 the Kim model $J_{c}\left(B_{z}\right)=J_{c}(0) B_{0} /\left(B_{0}+\left|B_{z}\right|\right)$ was considered, while in Ref. 22 the Kim model and the exponential dependence $J_{c}\left(B_{z}\right)=J_{c}(0) \exp \left(-\left|B_{z}\right| / B_{0}\right)$ were analyzed where $J_{c}(0)$ and $B_{0}$ are some constant parameters. Our results in the case $p>0$ are similar to the results in the papers. Equations $(15,16)$ show that both models 2122 may be interpreted as angular dependences of $j_{c}(\theta)$ with maxima at $\theta=\pi / 2$ if $B_{0}$ is of the order of $J_{c}(0)$.

The position $x=x_{0}$ of the flux front $\gamma_{0}$ as a function of the increasing applied field $H$ is shown in Fig. 4 for the same anisotropic strips. One realizes that for $p>0$ the penetration depth $a-x_{0}$ is smaller, and for $p<0$ larger, than for the isotropic case $p=0$. The isotropic case is shown twice in Fig. 4: The numerical result for $x_{0}$ obtained with a finite creep exponent $n=101$ is only slightly smaller than the ideal static result, Eq. (20). This demonstrates the accuracy of our computations.

Using the above results for the infinitely thin strip and equations of Sec. II, we can now describe the two dimen- sional critical state of the anisotropic strip with small but finite thickness.

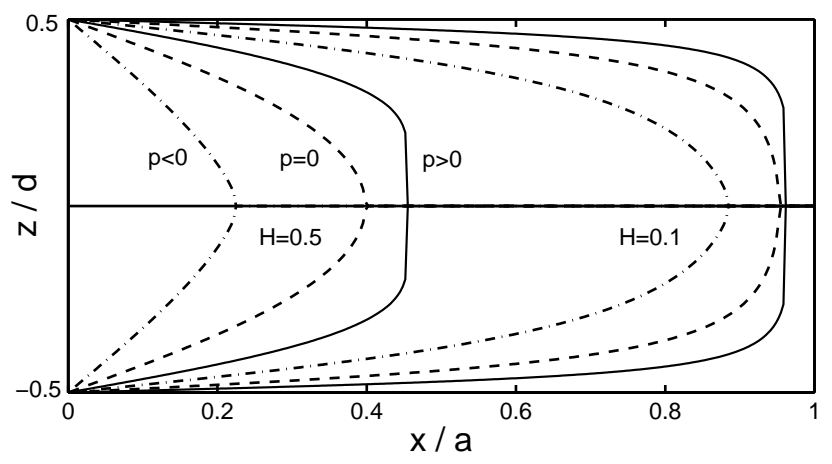

FIG. 5. Flux fronts $z_{\gamma}(x)$ in thin strips in increasing field $H$ calculated from Eq. (21) and the data $J(x)$ for isotropic pining ( $p=0$, dashed lines) and anisotropic pinning with $p=1, q=4$ (solid lines) and $p=-0.5,2$ (dash-dotted lines). Shown are two applied fields $H=0.1,0.5$ in units of $j_{c}(0) d$. Note the different scales for $x$ and $z$.

The general 3D flux front $\gamma$ (Fig. 1) is determined by Eq. (6) which is rewritten for our model Eqs. $(17,18)$ as

$$
z_{\gamma}(x, y)=\frac{d}{2}\left[1-\frac{J(x, y)}{J_{\gamma_{0}}}\right]
$$

where $J_{\gamma_{0}}=(1+p) j_{c}(0) d$ and $J(x, y)$ is the value of the sheet current at a given point inside $\gamma_{0}$. Fig. 5 shows $z_{\gamma}(x)$, the spatial profile or cross section of flux fronts $\gamma$, in thin strips with anisotropic pinning. Note that for $p>0$ the fronts have a wide flat part near the central plane $z=0$, while for $p<0$ the fronts have a more rounded shape near $x=x_{0}$ as compared to the isotropic case $p=0$.

Physically, the wide flat flux front for $p>0$ results from the fact that in this case the flux lines oriented perpendicularly to the film (and to the $\mathrm{CuO}$ planes) are less pinned and can thus penetrate more easily than the flux lines oriented parallel to the film plane. In the opposite case $p<0$, flux lines in the film plane move more easily and cause a more pointed wedge-like front.

The critical states of thick isotropic strips and disks were calculated in Refs. 23,24 using the appropriate 2D equations. The isotropic fronts $(p=0)$ obtained from Eq. (21) and depicted in Fig. 5 coincide with the flux fronts computed for such strips 23 and disks 24 if one takes $d \ll a$ and $j_{c}=j_{c}(0)$. These flux front profiles are equal for strips and disks since the appropriate static sheet currents $J(x)$ and $J(r)$ have identical forms 3 described by Eqs. $(19,20)$; for the disk $x$ should be replaced by the radial coordinate $r$ and $a$ means the disk radius. Interestingly, the thin film solution can well describe features of thick strips and disks up to quite large aspect ratios $d / 2 a \approx 0.2$ as long as the flux front is not too close to the center $\left(x_{0}>d\right)$. 

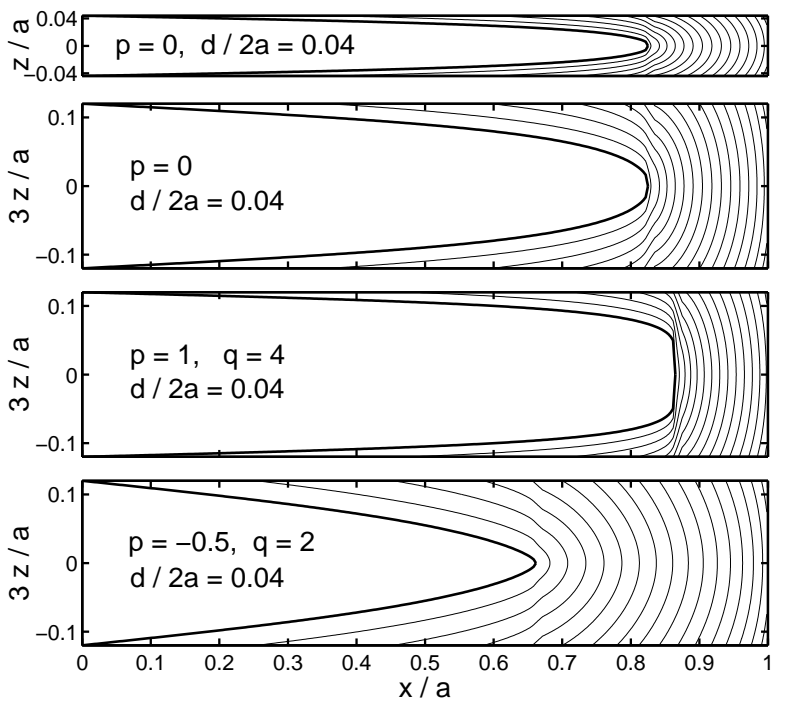

FIG. 6. Magnetic field lines in thin strips with aspect ratio $d / 2 a=1 / 25$ in a perpendicular magnetic field $H=0.2 j_{c}(0) d$. Isotropic pinning ( $p=0$, top) and same anisotropic pinning examples as in Figs. 3-5 are shown. The upper plot is to scale, the lower three plots are stretched along $z$ for clarity. The depicted lines are contours of the vector potential with nonequidistant levels $A_{i} \propto i^{2}, i=0,1,2,3, \ldots$, and are thus directed along the magnetic field. The nearly equidistant lines indicate a nearly constant gradient of the field magnitude. The inner contour $A(x, z)=0$ coincides with the flux front $\gamma$.

In a strip the sheet current has a fixed direction, and thus there is no rotation of the flux lines in the region inside $\gamma_{0}$, i.e., $\psi=$ const in Eq. (3). On the other hand, since in the considered case any in-plane anisotropy of pinning is absent, the flux lines are not twisted in the region $x \geq x_{0}$, and the solution (9-11) is valid. The distribution of $B_{t}=B_{x}(x, y, z)$ in the strip can be found using Eqs. $(5,11)$. For our model $(17,18)$, these equations are solved analytically, yielding for $x \leq x_{0}$

$$
\begin{array}{rrr}
B_{x}(x, z)=0, & |z| \leq z_{\gamma}(x), \\
\left|B_{x}(x, z)\right|=j_{c}(0)(1+p) & {\left[|z|-z_{\gamma}(x)\right],} \\
z_{\gamma}(x) \leq|z| \leq d / 2,
\end{array}
$$

while in the region $x \geq x_{0}$ we arrive at

$$
\begin{array}{r}
\left|B_{x}(x, z)\right|=j_{c}(0)|z|\left[1+p \exp \left(-\frac{q B_{z}(x) d}{2|z| B_{c r}}\right)\right], \\
|z| \leq d / 2 .
\end{array}
$$

Note that in the anisotropic case $(p \neq 0) B_{x}$ is not linear in $z$ in the region $x \geq x_{0}$. Taking into account these equations and the fact that $B_{z}$ is independent of $z$, one finds the $2 \mathrm{D}$ distributions of $\mathbf{B}$ inside the thin anisotropic strips shown in Fig. 6. As can be seen in the isotropic case our approach gives the expected 23 magnetic field lines except in a narrow region near the flux front, where this approximation breaks down (see Sec. II). The width of this region is less than, or of the order of $d$.

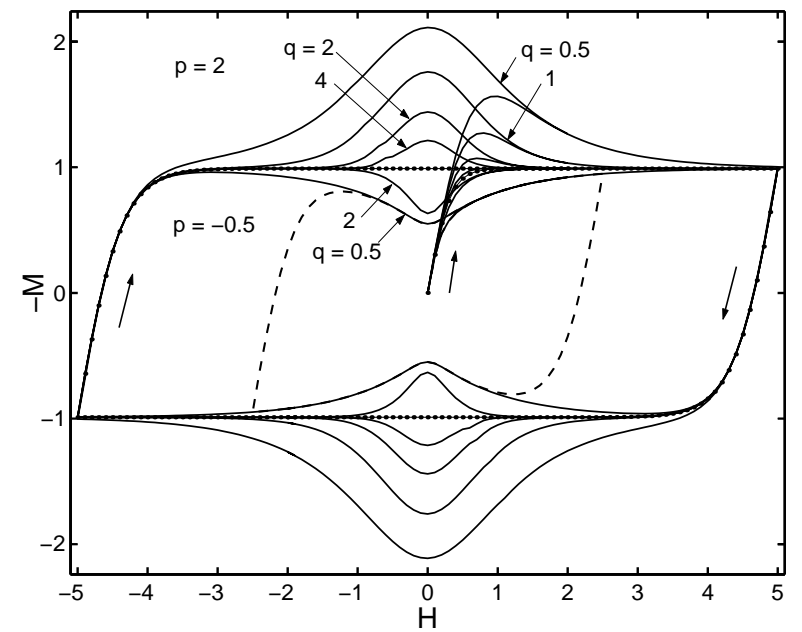

FIG. 7. Magnetization curves of thin strips with anisotropic pinning, Eqs. $(17,18)$. The dotted line is for isotropic pinning $(p=0)$. The loops with a hump are for $p=2$ and the loops with a dip for $p=-0.5$. The dashed curve has half the sweep amplitude. The applied field $H$ is in units of $j_{c}(0) d$ and $M$ is the magnetic moment per unit length in units of $j_{c}(0) d a^{2}$. Creep exponent $n=51$.

Knowing the direction of $\mathbf{B}(x, z)$ we can further calculate the distribution of $j=j_{c}$ in the region outside the 3D front $\gamma$ (inside $\gamma$ one has $j=0$ ). When $x<x_{0}$, the flux lines are practically parallel to the $x y$ plane, and thus $j_{c}=j_{c}(\pi / 2)=j_{c}(0)(1+p)$. If $x>x_{0}, j_{c}$ is given by the first equation (18) with $t$ replaced by the ratio $B_{z}(x) /\left[\mu_{0} j_{c}(0) z\right]$. Note that for $p>0$ and $x>x_{0}$, $j_{c}(x, z)$ is greater near the surfaces $(|z| \approx d / 2)$ than in the central plane of the strip $(z=0)$. The opposite situation occurs when $p<0$.

In Fig. 7 the virgin magnetization curves and magnetic hysteresis loops $M(H)$ are presented for strips with various anisotropies of pinning. For $a b$ plane pinning $(p>0)$ a so-called central peak appears. Thus in high- $T_{c}$ superconductors this often observed peak 25 in principle may be caused by intrinsic pinning by the $\mathrm{CuO}$ planes 17 Note that in this case the width of the peak is proportional to the thickness $d$ of the sample (our field units are $j_{c}(0) d$ ). From this one may estimate the contribution of the intrinsic pinning when the peak is observed. In the opposite case $p<0$, i.e. when $j_{c}(\theta)$ has a minimum at $\theta=\pi / 2$, a dip in $|M(H)|$ occurs in the region of weak fields. An additional dependence of $j_{c}$ on $|\mathbf{B}|$ may then lead to a so-called fishtail (or peak) effect 11 with a maximum of $|M|$ at some intermediate field. In Fig. 7 the zig-zag sweep of the applied field $H(t)$ has constant ramp rate, $|d H / d t|=1$ in units where $j_{c}(0) d=a=\mu_{0}=E_{0}=1$ in the used current-voltage law $E(J)=E_{0}\left(J / J_{c}\right)^{n}$ with $n=51$, cf. Appendix B. When the creep exponent $n$ is lowered to a more realistic value $n=11$ measured e.g. in Ref. 26, the amplitudes of the peak and dip slightly 
decrease, and the sharp edges of $M(H)$ at the largest and lowest $H_{a}$ are rounded, see also the figures in Refs. 19d d, 23, 24.

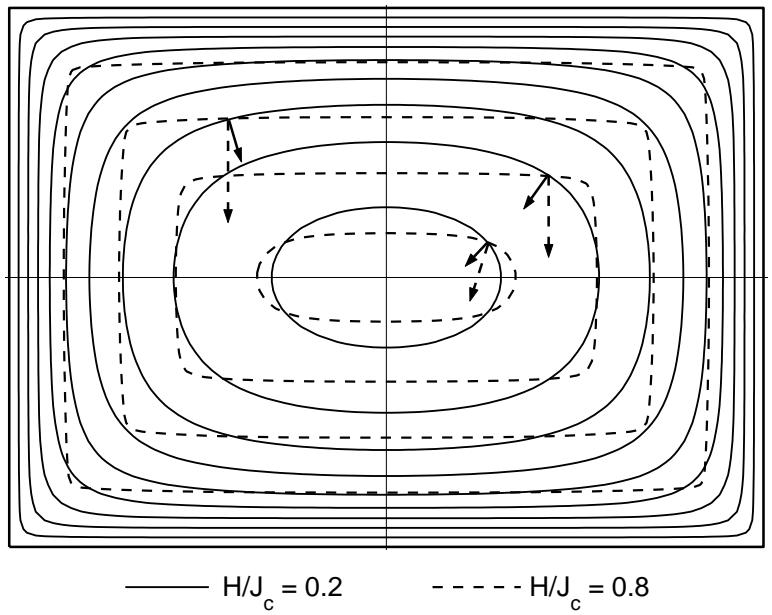

FIG. 8. Stream lines of the sheet current in a thin rectangular platelet with side ratio 1.4 and isoptropic pinning. Bean model $J_{c}=$ const. Applied field $H / J_{c}=0.2$ (solid lines) and $H / J_{c}=0.8$ (dashed lines). Creep exponent $n=21$. The arrows perpendicular to the stream lines and of length proportional to the sheet current, indicate the direction of the flux lines lying at different depths.

\section{THIN RECTANGULAR PLATES}

The critical state of rectangular platelets is qualitatively similar to that of the strip. In particular, for aspect ratios $b / a>1.4$ (see Figs. $1,8,9)$ the profiles $J_{y}(x, 0)$ and $B_{z}(x, 0)$ along the shorter axis are practically identical to the profiles in long strips with $b / a \rightarrow \infty$. There are, however, several qualitative differences as compared to the magnetic behavior of long strips or circular disks:

a) In platelets with shape different from strips or circular disks, the stream lines of the sheet current $\mathbf{J}(x, y)=$ $\left(J_{x}, J_{y}\right)$ do no longer coincide with the contour lines of $B_{z}(x, y)$ and the penetrating flux fronts $\gamma_{0}$ (Fig. 1). This new feature is described in detail in Ref. 4 for films with elliptical shape.

b) In increasing applied field $H$, the direction of the sheet current $\mathbf{J}(x, y)$ changes with $H$ at any given point $(x, y)$ positioned inside the front $\gamma_{0}$ and away from the symmetry axes $x=0, y=0$, or edges $x= \pm a, y= \pm b$. In the isotropic case this rotation of $\mathbf{J}(x, y)$ comes to a halt when the flux front passes through the point.

Both features a) and b) occur only in perpendicular geometry. In long rods with the same rectangular cross section in longitudinal field, the $B_{z}$ contours, $\mathbf{J}$ stream lines, and flux fronts are concentric rectangles, and inside the flux front both $B_{z}(x, y)$ and $\mathbf{J}(x, y)$ vanish. From the feature b) follows a further interesting effect:
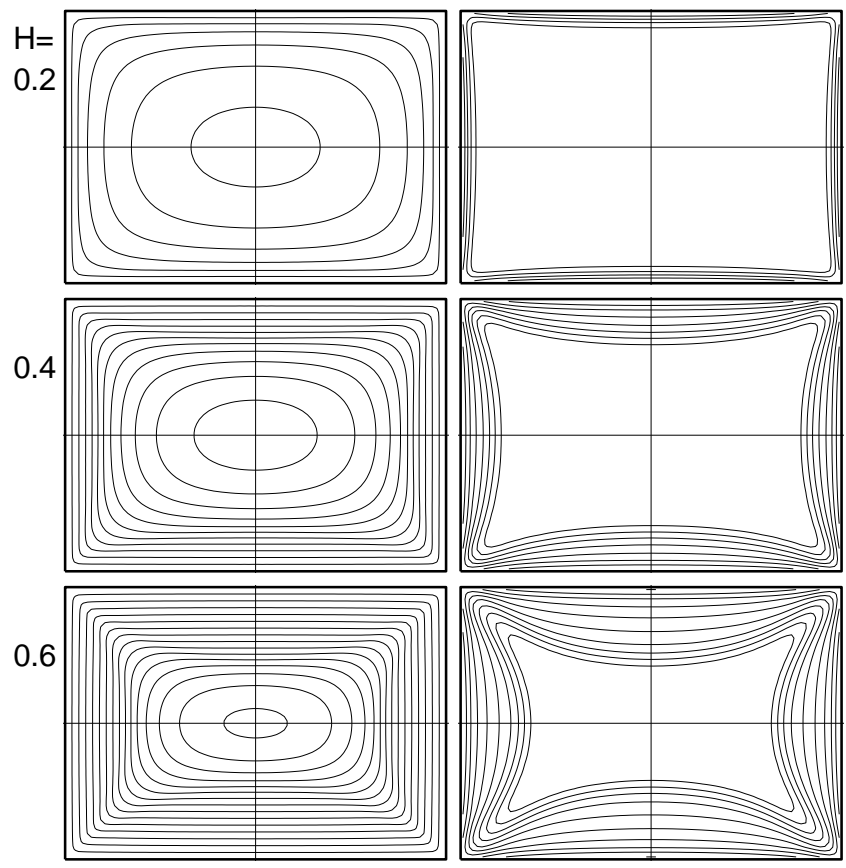

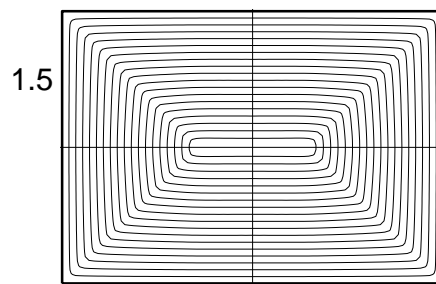

current stream lines

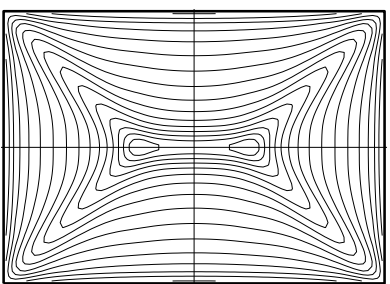

contours of $B_{z}(x, y)$
FIG. 9. Stream lines of the sheet current $\mathbf{J}(x, y)$ (left) and contour lines of the perpendicular magnetic induction $B_{z}(x, y)$ (right) in a thin rectangular plate with out-of-plane anisotropic pinning and side ratio 1.4. The anisotropy $j_{c}(\theta)$ is described by the model Eqs. $(17,18)$ with $p=2$ and $q=4$. The applied magnetic field is (from top to bottom) $H=0.2$, $0.4,0.6,1.5$ in units of $j_{c}(0) d$. Creep exponent $n=21$.

In thin rectangles a rotation of the penetrating flux lines may occur inside the front $\gamma_{0}$. In this region the flux lines surround a thin flat field-free core and are nearly parallel to the $x, y$ plane. However, at a given point $(x, y)$ the flux lines lying at different $z$ can have different in-plane orientations. This rotation of flux lines is due to the rotation of the direction of the sheet current and occurs in any isotropic or anisotropic thin superconductor with shape different from a strip or circular disk. An example of this rotation is shown in Fig. 8.

The stream lines of the sheet current and the contours of $B_{z}(x, y)$ in a thin rectangular plate with strong outof-plane anisotropy of pinning are depicted in Fig. 9 for increasing applied field $H$. The stream lines are contours of the function $g(x, y)$ introduced in Appendix B. In all contour plots in Fig. 9 the same constant level spacing is used: $\Delta g=1 / 20$ and $\Delta B_{z}=1 / 10$ in units where $j_{c}(0) d=a=\mu_{0}=1$. In our computation based on 
Ref. 母 we used $|d H / d t|=1$ and $E_{0}=1$ and $n=51$ in $E=E_{0}\left(J / J_{c}\right)^{n}$. The shape of the depicted lopis is very similar to their shape in the isotropic plate, 1 U i.e. different $J_{c}\left(B_{z}\right)$ dependences lead to almost the same penetration pattern. This is so since the shape of these loops is determined mainly by the specimen shape: At small $H \ll J_{c}(0)$, one has almost complete field expulsion and the stream lines are thus independent of any material property, being rectangular near the edges and circular near the center of the rectangle. After the flux front has passed through a given point, $J$ abruptly tends to nearly a constant, see Fig. 3 and Eq. (17). The situation then becomes similar to the isotropic case, and the stream lines practically coincide with concentric rectangles with constant spacing proportional to $1 / \mathrm{J}$.

Since the current stream lines in the rectangular platelet do not coincide with the lines $B_{z}=$ const, and $J$ depends on $B_{z}$, the penetration pattern in the anisotropic case could differ from that obtained in the isotropic superconductor. 1 . However, even a close look can hardly discover a qualitative difference in the shapes of the contours in isotropic and strongly out-of-plane anisotropic (Fig. 9) rectangular plates, in stark contrast to the situation with in-plane-anisotropy 8 A minor difference is that in the partly penetrated state $(H=0.4$ and $H=0.6$ in Fig. 9) the stream lines outside the flux front and near the axes $x=0$ and $y=0$, are slightly convex towards the center, while for isotropic Bean pinning the stream lines are straight there (or are slightly concave due to the finite creep exponent $n$ ). As expected from the strip results, Fig. 3, with out-of-plane pinning the density of the sheet-current stream-lines near the flux front is larger (when $p>1$ ) or smaller (when $p<1$ ) as compared to the case of isotropic pinning $(p=0)$, cf. Eq. (17). The same statement is true for the contours of $B_{z}$ near the front.

Figure 10 shows magnetization curves of thin rectangular plates with side ratio 1.4 and various out-of-plane anisotropies. These magnetization loops exhibit central peaks or dips caused by the anisotropy and are very similar to those shown in Fig. 7 for strips. Thus, one can conclude that the features of the loops at $H \sim j_{c} d$ are specified mainly by the anisotropy of flux-line pinning (i.e., by $p$ and $q$ ) rather than by the shape of the sample. This property should be useful for determining these pinning parameters from experiments.

\section{CONCLUSIONS}

In many papers thin superconductors are considered as $2 \mathrm{D}$ samples with a constant critical sheet current $J_{c}$ or with $J_{c}\left(B_{z}\right)=j_{c}\left(B_{z}\right) d$ if $j_{c}$ depends on the magnitude of the magnetic induction. However, when an out-ofplane anisotropy of pinning exists, the current density also depends on the angle $\theta$ between $\mathbf{B}$ (the flux-line direction) and the normal to the sample plane (our $z$ axis), and thus the critical state problem becomes threedimensional. We have shown here that the general 3D critical state problem for thin superconductors of arbitrary shape and with arbitrary 3D anisotropy of pinning can be separated into a $1 \mathrm{D}$ problem, which treats the current density and induction across the thickness of the sample, and the 2D problem of thin superconductors with a new induction dependence of the critical sheet current $J_{c}\left(B_{z}\right)$ flowing in the plane of the superconductor. The new $J_{c}\left(B_{z}\right)$ dependence is determined by the out-of-plane anisotropy of the critical current density $j_{c}(\theta)$ and modifies the original $B_{z}$ dependence and in-plane anisotropy of $J_{c}$, if they exist. The resulting $2 \mathrm{D}$ thin film problem with given $J_{c}\left(B_{z}\right)$ can be solved by standard numerical and analytical methods. 1824 Our theory generalizes the approach of Ref. 10 for a circular disk.

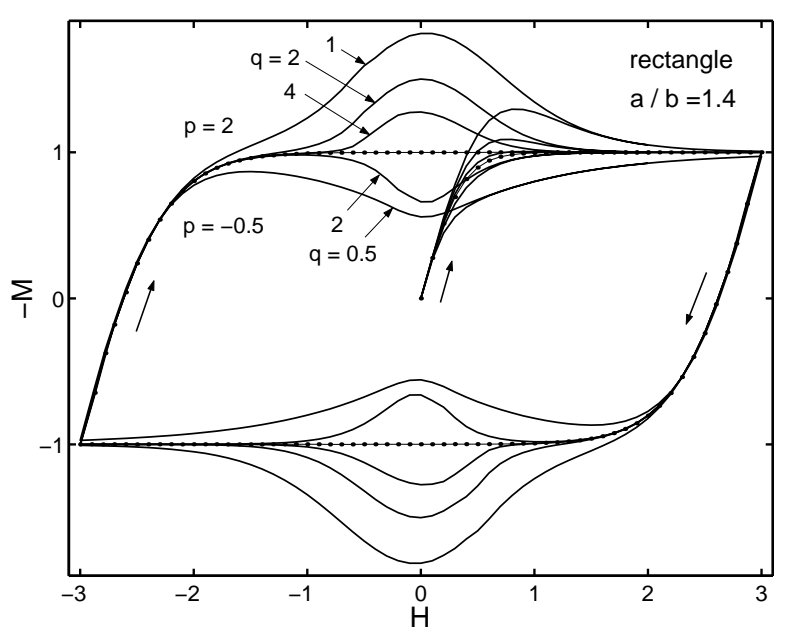

FIG. 10. Magnetization curves of thin rectangular plates with anisotropic pinning, Eqs. $(17,18)$, side ratio $a / b=1.4$, and $n=51$. As in Fig. 7, the dotted line is for isotropic pinning $(p=0)$, the loops with a hump are for $p=2$, and the loops with a dip for $p=-0.5$. The applied field $H$ is in units of $j_{c}(0) d$ and $M$ is the magnetic moment in units of the saturation moment of the rectangle, $(2 / 3) j_{c}(0) d b^{2}(3 a-b)$.

Knowing the solutions of these 1D and 2D problems, the critical state in three-dimensional thin flat superconductors (Fig. 1) can be understood. Two qualitatively new features of the critical state occur in this general case as compared to the highly symmetric cases of isotropic disks or strips, which are commonly considered. First, when the shape of the sample differs from a strip or a disk then a rotation of flux lines (differently oriented in different planes $z=$ const) may occur in the region where $B_{z}=0$, i.e. where the flux lines surround a nonpenetrated flat core and are nearly parallel to the sample plane since the sample is thin. Second, if both out-ofplane and in-plane anisotropies of pinning exist, the flux lines even in thin superconductors are not only curved but also twisted in the region where $B_{z} \neq 0$, i.e. outside 
the front line $\gamma_{0}$ in Fig. 1. Twist means that the in-plane component of the direction of the flux lines at a given point $x, y$ changes with the depth $z$.

Using the above approach, the critical states of strips and rectangular platelets with only out-of-plane anisotropy are analyzed. We consider a rather general analytical model (18) for this anisotropy, which can simulate both the intrinsic pinning by $\mathrm{CuO}$ planes and pinning by extended defects aligned with the $c$ axis in high$T_{c}$ superconductors. We obtain the shape of the flux front and the two-dimensional distributions of the magnetic induction and current density for the anisotropic strip. In the case of intrinsic pinning, the flux front has a wide flat part near the central plane, Fig. 5; the current density peaks near this flat front, and the induction profile is very steep there, see Fig. 3. In fact, a so-called current string 27 occurs in this case, i.e., an additional current flows along this steep front as compared to the isotropic situation. In Refs. 27.28 the string was considered in connection with a finite lower critical field $H_{c 1}$. However, in the considered case the string is not due to a finite $H_{c 1}$ but results from the anisotropy of pinning, and the jump of $B_{z}$ at the flux front considerably exceeds $H_{c 1}$ since $H_{c r}=j_{c} d / 2 \gg H_{c 1}$ was assumed above. This type of out-of-plane anisotropy leads to a central peak in the magnetization curve, Fig. 7.

A different situation occurs when the $\theta$ dependence of $j_{c}$ has a minimum at $\theta=\pi / 2$. In this case the flux front has the shape of a wedge with a rounded point that becomes sharper with increasing anisotropy. As can be seen in Figs. 3b,c the current density near the front line $\gamma_{0}$ now has no peak and no sharp step (as for isotropic pinning) but it decreases monotonically and has only a rounded step and an inflection point with vertical slope at this front line. The induction $B_{z}$ now vanishes less steeply than in the isotropic case, and in the limit of large such anisotropy the induction profile near the front decreases almost linearly. The magnetization loop for this type of anisotropy has a central dip, see Fig. 7.

All these features remain qualitatively the same when a smaller creep exponent $n$ is chosen in $E \propto J^{n}$, Eq. (B6), or when the superconductor has a rectangular shape. In particular, with smaller $n$ the cusp in $J(x)$ remains sharp and the profile of $B_{z}(x)$ remains steep, see Fig. $3 \mathrm{~d}$ with $n=11$. As shown in Figs. 7 and 10, the magnetization loops of thin rectangular plates with out-of-plane anisotropy exhibit the same central peak or dip as thin strips.

For any given dependence $j_{c}(\mathbf{B})$, the equations of this paper enable one to compute various characteristics of the critical state in thin superconducting samples of realistic shapes. With this theory at hand, experimental investigation of flux-density profiles, of the $H$ dependence of the penetrating flux front (e.g., by magneto-optics), and of magnetization loops, will yield information not only on the strengh but also anisotropy of flux-line pinning in superconductors.

\section{ACKNOWLEDGMENTS}

G.P.M. acknowledges the hospitality of the MaxPlanck-Institute für Metallforschung, Stuttgart.

\section{APPENDIX A: ELECTRIC FIELD IN ANISOTROPIC SUPERCONDUCTORS}

In a uniform anisotropic superconductor the direction of the electric field $\mathbf{E}$ generated by vortex motion in general will not coincide with the direction of the current. To study this effect, we start with the situation when a current with the density $j$ flows in the plane normal to the magnetic induction $\mathbf{B}$ (below we call this plane the $N$ plane). The creep activation barrier $U\left[B, \varphi, j / j_{0}(B, \varphi)\right]$ is assumed to vanish at $j=j_{0}(B, \varphi)$ [this is the definition of the factor $\left.j_{0}(B, \varphi)\right]$. Here the angle $\varphi$ specifies the direction of the current in the $N$ plane, $\mathbf{j} \propto(\cos \varphi, \sin \varphi)$, and $U\left[B, \varphi, j / j_{0}(B, \varphi)\right]$ is the value of the barrier which a flux bundle has to overcome to hop in the direction of the Lorentz force, i.e., along the vector $[\mathbf{j} \times \mathbf{B}]$. At given temperature $T$, the expression $\exp \{-U[B, \zeta, j \cos (\zeta-$ $\left.\left.\varphi) / j_{0}(B, \zeta)\right] / T\right\}$ gives the probability for a flux-bundle to jump in the direction normal to any given in-plane vector $(\cos \zeta, \sin \zeta)$. Hence, the electric field $\mathbf{E}$ is obtained by averaging this expression over all angles $\zeta$ in the interval $\varphi-\pi / 2 \leq \zeta \leq \varphi+\pi / 2$ (we disregard fluxbundle jumps against the Lorentz force). If the effective depth of the flux-pinning well $[\partial U(B, \varphi, x) / \partial x]_{x=1}$ considerably exceeds $T$, the direction of $\mathbf{E}$ in the $N$ plane, $\left(\cos \zeta_{0}, \sin \zeta_{0}\right)$, can be found as the position of the minimum of $U\left[B, \zeta, j \cos (\zeta-\varphi) / j_{0}(B, \zeta)\right]$ with respect to variations of $\zeta$. Since, according to the definition of $j_{0}(B, \zeta)$, one has $\partial U(B, \zeta, 1) / \partial \zeta=0$, one obtains the following formula for $\zeta_{0}$ in the critical state:

$$
\tan \left(\zeta_{0}-\varphi\right)=-\frac{j_{0}^{\prime}\left(B, \zeta_{0}\right)}{j_{0}\left(B, \zeta_{0}\right)}
$$

where $j_{0}^{\prime}(B, \zeta) \equiv \partial j_{0}(B, \zeta) / \partial \zeta$. As for the critical current density $j_{c}(B, \varphi)$, we have

$$
j_{c}(B, \varphi)=\frac{j_{0}\left(B, \zeta_{0}\right)}{\cos \left(\zeta_{0}-\varphi\right)}=\left[j_{0}\left(B, \zeta_{0}\right)^{2}+j_{0}^{\prime}\left(B, \zeta_{0}\right)^{2}\right]^{1 / 2} .
$$

It is also useful to express the angle $\zeta_{0}$ in terms of $j_{c}(B, \varphi)$, i.e., the quantity determined in experiments,

$$
\delta \equiv \zeta_{0}-\varphi=-\arctan \left\{\frac{j_{c}^{\prime}(B, \varphi)}{j_{c}(B, \varphi)}\right\},
$$

where $j_{c}^{\prime}(B, \varphi)=\partial j_{c}(B, \varphi) / \partial \varphi$. The above formulas have been derived under the assumption that $\cos (\zeta-$ $\varphi) / j_{0}\left(B_{z}, \zeta\right)$ has only one maximum in the interval $\varphi-$ $\pi / 2<\zeta<\varphi+\pi / 2$. This situation does not always occur in twinned crystals. In such crystals the angle $\zeta_{0}$ may 
have a fixed value in some interval of angles $\varphi$, and thus $j_{c}(\varphi)=j_{c}\left(\zeta_{0}\right) / \cos \left(\zeta_{0}-\varphi\right)$ inside the interval. However, note that Eq. (A1) is still valid in this case.

We now present the formulas for the case considered in Sec. II: critical currents flow in the $x y$ plane with $\mathbf{j}_{c}=\left(j_{c} \cos \varphi, j_{c} \sin \varphi\right)$, and a flux-line element has the direction $(\sin \theta \cos \psi, \sin \theta \sin \psi, \cos \theta)$. Since in the absence of flux cutting only the component of $\mathbf{j}_{c}$ normal to B is essential, the critical current density in the $N$ plane, $j_{c}^{\perp}$, has the form

$$
j_{c}^{\perp}\left(B_{z}, B_{t}, \varphi-\psi, \psi\right)=n_{\perp}(\varphi) j_{c}\left(B_{z}, B_{t}, \varphi-\psi, \psi\right),
$$

where $n_{\perp}(\varphi)=\left[1-\cos ^{2}(\varphi-\psi) \sin ^{2} \theta\right]^{1 / 2}$. It follows from Eq. (A1) that the angle $\delta$ between $\mathbf{E}$ and $\mathbf{j}_{c}^{\perp}$ is given by the formula

$$
\tan \delta=-\frac{\partial\left[\ln j_{c}^{\perp}\left(B_{z}, B_{t}, \varphi-\psi, \psi\right)\right]}{\partial \varphi} \cdot \frac{n_{\perp}^{2}(\varphi)}{\cos \theta} .
$$

The unit vector e directed along $\mathbf{E}$ has the components:

$$
\begin{array}{r}
e_{x}=\frac{1}{n_{\perp}(\xi)}\left[\cos \xi-\sin ^{2} \theta \cos \psi \cos (\xi-\psi)\right], \\
e_{y}=\frac{1}{n_{\perp}(\xi)}\left[\sin \xi-\sin ^{2} \theta \sin \psi \cos (\xi-\psi)\right], \\
e_{z}=-\frac{1}{n_{\perp}(\xi)} \sin \theta \cos \theta \cos (\xi-\psi),
\end{array}
$$

where the angle $\xi$ is defined by the expression

$$
\tan (\xi-\psi)=\cos \theta \frac{\tan (\varphi-\psi)+\cos \theta \tan \delta}{\cos \theta-\tan \delta \tan (\varphi-\psi)}
$$

In other words, e is the normalized projection of the vector $(\cos \xi, \sin \xi)$ onto the $N$ plane. This explains the meaning of the angle $\xi$.

It is important to keep in mind one special case. If an in-plane anisotropy of flux-line pinning is absent, it follows from symmetry considerations that at fixed $B_{z}, B_{t}$, $\psi$ the derivatives $\partial j_{c}^{\perp} / \partial \varphi$ and $\partial j_{c} / \partial \varphi$ are equal to zero when $\varphi-\psi= \pm \pi / 2$. Thus, in this case one has $\delta=0$, $\xi=\varphi$, and the directions of $\mathbf{E}, \mathbf{j}_{c}$ and $\mathbf{j}_{c}^{\perp}$ coincide.

\section{APPENDIX B: DYNAMIC EQUATIONS}

It is convenient to express the sheet current in the film through a scalar function $g(x, y)=g(\mathbf{r})$ as

$$
\mathbf{J}(\mathbf{r})=\nabla \times \hat{\mathbf{z}} g(\mathbf{r})=-\hat{\mathbf{z}} \times \nabla g(\mathbf{r}) .
$$

This substitution guarantees that $\nabla \cdot \mathbf{J}=0$ and that the current flows along the specimen boundary $\Gamma$ if one puts $g(\mathbf{r})=$ const $=0$ there (the lines $g(\mathbf{r})=$ const coincide with the current stream lines). Then, Eq. (14) is transformed as follows, 29

$$
\frac{B_{z}(\mathbf{r})}{\mu_{0}}=H+C(\mathbf{r}) g(\mathbf{r})-\int_{S} \frac{g\left(\mathbf{r}^{\prime}\right)-g(\mathbf{r})}{4 \pi R^{3}} d^{2} r^{\prime},
$$

with

$$
4 \pi C(\mathbf{r})=\int_{\bar{S}} \frac{d^{2} r^{\prime}}{R^{3}}=\int_{0}^{2 \pi} \frac{d \phi}{R(\phi)} .
$$

The integral in Eq. (B1) is taken in the Cauchy sense. In formulas (B2) the integration area $\bar{S}$ is the entire $x, y$ plane with exclusion of the sample area $S ; R(\phi) \equiv|\mathbf{s}-\mathbf{r}|$; $\mathbf{s}$ defines the position of a point on the boundary $\Gamma$; and $\phi$ is the angle of the vector $\mathbf{s}-\mathbf{r}$ relative to an arbitrary fixed direction. Eq. (B1) admits inversion,

$$
g(\mathbf{r})=\int_{S} K\left(\mathbf{r}, \mathbf{r}^{\prime}\right)\left[\mu_{0}^{-1} B_{z}\left(\mathbf{r}^{\prime}\right)-H\right] d^{2} r^{\prime},
$$

where the kernel $K\left(\mathbf{r}, \mathbf{r}_{1}\right)$ is found from the following equation,

$$
\begin{gathered}
K\left(\mathbf{r}, \mathbf{r}_{1}\right)=\frac{1}{C(\mathbf{r})}\left\{\delta\left(\mathbf{r}-\mathbf{r}_{1}\right)\right. \\
\left.+\int_{S} \frac{K\left(\mathbf{r}^{\prime}, \mathbf{r}_{1}\right)-K\left(\mathbf{r}, \mathbf{r}_{1}\right)}{4 \pi R^{3}} d^{2} r^{\prime}\right\},
\end{gathered}
$$

which can be solved by iteration starting with $K\left(\mathbf{r}, \mathbf{r}_{1}\right)=$ 0 .

We now differentiate Eq. (B3) with respect to time $t$, take into account the Maxwell equatiph, $\partial B_{z} / \partial t=$ $-\hat{\mathbf{z}} \cdot[\nabla \times \mathbf{E}]$, and the current-voltage law, 30$]$

$$
\mathbf{E}=\mathbf{E}\left(\mathbf{J}, B_{z}\right),
$$

which expresses the electric field $\mathbf{E}$ through the sheet current J. Then, we arrive at an equation for $g(\mathbf{r})$,

$$
\begin{gathered}
\frac{\partial g(\mathbf{r}, t)}{\partial t}=\int_{S} K\left(\mathbf{r}, \mathbf{r}^{\prime}\right) \\
\left\{-[\hat{\mathbf{z}} \times \nabla] \cdot \mathbf{E}\left[-\hat{\mathbf{z}} \times \nabla g\left(\mathbf{r}^{\prime}, t\right), B_{z}\left(\mathbf{r}^{\prime}\right)\right]-\frac{\partial H(t)}{\partial t}\right\} d^{2} r^{\prime} .
\end{gathered}
$$

Equations (B1) and (B5) allow one to find $B_{z}(\mathbf{r}, t), g(\mathbf{r}, t)$ [and thus $\mathbf{J}(\mathbf{r}, t)$ ] if the prehistory $H(t)$ and the currentvoltage law are given.

The static critical state can be calculated by a dynamic approach using any model law $\mathbf{E}$ which has a sharp bend at $J=J_{c}$. The specific form of this dependence is irrelevant; e.g. we may use the power law

$$
\mathbf{E}=\mathbf{E}_{0}\left(\frac{|\mathbf{J}|}{J_{c}}\right)^{n}
$$

and take the limit $n \gg 1$. That the static critical state is indeed reached in the limit $n \rightarrow \infty$ was shown rigorously in Ref. 31. The function $J\left(B_{z}, \varphi_{0}\right)$ resulting from Eq. (12) or from the appropriate generalization of this equation should be used as the critical sheet current $J_{c}$ in Eq. (B6) for currents with direction $\left(\cos \varphi_{0}, \sin \varphi_{0}\right)$. The direction of the constant vector $\mathbf{E}_{0}$ in general does 
not coincide with $\mathbf{J}$ if there is anisotropy of flux-line pinning in the $x y$ plane (see Appendix A). At given $\varphi_{0}$, the angle $\xi_{0}$ defining the direction of $\mathbf{E} \propto\left(\cos \xi_{0}, \sin \xi_{0}\right)$ is determined by the formula

$$
\tan \left(\xi_{0}-\varphi_{0}\right)=-\frac{\partial\left[\ln J\left(B_{z}, \varphi_{0}\right)\right]}{\partial \varphi_{0}} .
$$

Thus, to describe the critical state of an infinitely thin superconductor, it is sufficient to solve the dynamical Eqs. $(\mathrm{B} 1, \mathrm{~B} 5, \mathrm{~B} 6)$ with the initial condition $\left.g(x, y)\right|_{t=0}=$ 0 . This dynamical approach appears to be more convenient than solving the static Eqs. $(13,14)$ with an initially unknown flux-front $\gamma_{0}$.

${ }^{1}$ E. H. Brandt, Rep. Prog. Phys. 58, 1465 (1995).

${ }^{2}$ P.N. Mikheenko and Yu.E. Kuzolev, Physica C 204, 229 (1994).

${ }^{3}$ E.H. Brandt, M.V. Indenbom and A. Forkl, Europhys. Lett. 22, 735 (1993); E.H. Brandt and M.V. Indenbom, Phys. Rev. B 48, 12893 (1993); E. Zeldov, J.R. Clem, M. McElfresh and M. Darwin, Phys. Rev. B 49, 9802, (1994).

${ }^{4}$ G.P.Mikitik and E.H. Brandt, Phys. Rev. B 60, 592 (1999).

${ }^{5}$ E.H. Brandt, Phys. Rev. Lett. 74, 3025 (1995).

${ }^{6}$ L. Prigozhin, J. Comp. Phys. 144, 180 (1998).

${ }^{7}$ E.H. Brandt, Phys. Rev. B 52, 15442 (1995).

8 Th. Schuster, H. Kuhn, E.H. Brandt, and S. Klaumünzer, Phys. Rev. B 56, 3413 (1997).

${ }^{9}$ M. Tachiki and S. Takahashi, Solid State Commun. 70, 2991 (1991).

${ }^{10}$ I.M. Babich and G.P. Mikitik, Phys. Rev. B 54, 6576 (1996).

${ }^{11}$ I.M. Babich and G.P. Mikitik, Pis'ma Zh. Eksp. Teor. Fiz. 64, 538 (1996) [JETP Lett. 64, 586 (1996)]; I.M. Babich and G.P. Mikitik, Phys. Rev. B 58, 14207 (1998).

${ }^{12}$ E. Zeldov, A. I. Larkin, V. B. Geshkenbein, M. Konczykowski, D. Majer, B. Khaykovich, V. M. Vinokur, and H. Strikhman, Phys. Rev. Lett. 73, 1428 (1994); M. Benkraouda and J. R. Clem, Phys. Rev. B 53, 5716 (1996); R. Labusch and T. B. Doyle, Physica C 290, 143 (1997); T. B. Doyle, R. Labusch, and R. A. Doyle, Physica C 290, 148 (1997); E. H. Brandt, Phys. Rev. B 59, 3369 (1999); ibid, Phys. Rev. B 60, 11939 (1999).
13 To be able to consider the superconductor as a uniform medium, the characteristic distance between the twin boundaries, $l_{t w}$, should be less than the scale over which the currents and the fields are averaged, i.e., at least $l_{t w} \ll d$.

${ }^{14}$ A. M. Campbell and J. E. Evetts, Adv. Phys. 72, 199 (1972).

15 J. R. Clem, Phys. Rev. B 26, 2463 (1982); J. R. Clem and A. Perez-Gonzalez, Phys. Rev. B 30, 5041 (1984); A. Perez-Gonzalez and J. R. Clem, Phys. Rev. B 31, 7048 (1985).

${ }^{16}$ E. H. Brandt, Phys. Lett. 79A, 207 (1980); E. H. Brandt, J. Low Temp. Physics 39, 41 (1980); E. H. Brandt, J. Low Temp. Physics 44, 33, 59 (1981).

${ }^{17}$ L. W. Conner, A. P. Malozemoff, and I. A. Campbell, Phys. Rev. B 44, 403 (1991).

${ }^{18}$ E. H. Brandt, Phys. Rev. B 49, 9024 (1994).

${ }^{19}$ E. H. Brandt, Phys. Rev. B 50, 4034 (1994); Th. Schuster et al., Phys. Rev. Lett. 73, 1424 (1994); E. H. Brandt, Physica C 235-240, 2939 (1994); Phys. Rev. B 55, 14513 (1997).

${ }^{20}$ G. P. Mikitik and E. H. Brandt, Phys. Rev. B (submitted).

${ }^{21}$ J. McDonald and J. R. Clem, Phys. Rev. B 53, 8643 (1996).

${ }^{22}$ D. V. Shantsev, Y. M. Galperin, and T. H. Johansen, Phys. Rev. B 60, 13112 (1999).

${ }^{23}$ E. H. Brandt, Phys. Rev. B 54, 4246 (1996).

${ }^{24}$ E. H. Brandt, Phys. Rev. B 58, 6506 (1998).

${ }^{25}$ See, e.g., D. V. Shantsev, M. R. Koblischka, Y. M. Galperin, T. H. Johansen, L. Pust, and M. Jirsa, Phys. Rev. Lett. 82, 2947 (1999) and references therein.

${ }^{26}$ B. J. Jönsson-Åkerman, K. V. Rao, and E. H. Brandt, Phys. Rev. B 60, 14913 (1999).

${ }^{27}$ M. V. Indenbom, Th. Schuster, H. Kuhn, H. Kronmüller, T. W. Li, A. A. Menovsky, Phys. Rev. B 51, 15484 (1995).

${ }^{28}$ E. H. Brandt, Phys. Rev. B 59, 3369 (1999).

${ }^{29}$ E. H. Brandt, Phys. Rev. B 46, 8628 (1992).

${ }^{30}$ Note that we replace the current density $\mathbf{j}$ by the sheet current $\mathbf{J}$ in the original current-voltage law $\mathbf{E}\left(\mathbf{j}, B_{z}\right)$. This is justified by the following considerations. First, in this paper the dynamical approach is only a useful trick which allows us to obtain the solution of the critical state equations. Second, in the leading order in $d / L$, the electric field $\mathbf{E}(x, y, z)$ is a constant across the thickness of the sample in the region outside the flux front [this estimate is also confirmed by the result of computations for strips and disks of a finite thickness.23 24 Thus, if one replaces $j / j_{c}$ by $J / J_{c}$ in the current-voltage law, this is really a good approximation in solving the dynamic equations for thin superconductors.

31 J. W. Barrett and L. Prigozhin, Nonlinear Analysis. Theory, Methods and Applications (in print) (2000). 